\title{
Objetivos, indicaciones y modalidades de desintoxicación de los alcohólicos***
}

\author{
Paille, F.; Bazot, M.; Favre, J.D.*** \\ * Conclusiones y recomendaciones del grupo de trabajo francés para elaborar un documento de consenso. \\ ** Grupo de trabajo: BAZOT M, (presidente), BOUIX JC, CASAMITJANA F, CHEVREUL JP, COUZIGOU P, CRESPO G, DEMAZIERE J, FUSCIARDI J, \\ HURARD MC, MANTZ JM, MICHAUD P, OLIE JP, PAU G, PELLEGRI C, RANDU J, ROUGERON-JOBIN D, WAHL D \\ *** Société Française d'Alcoologie.
}

Enviar correspondencia:

J.D. Favre. Societé Française d'Alcoologie. 101, Av. Henri Barbuse F-92141 Clamart Cedex (FRANCIA)

\section{Resumen :}

La desintoxicación del alcohólico sólo tiene sentido dentro de una estrategia que supone tres etapas:

1) diagnóstico: Es importante separar, aunque en la práctica a veces no es fácil, el abuso del alcohol de la dependencia del alcohol, sobre todo desde que desde 1994 los síntomas físicos de abstinencia no son necesarios para el diagnóstico.

2) Todo cuadro de dependencia alcohólica confirmado justifica una desintoxicación terapéutica completa y prolongada, integrada dentro de un proyecto de vida en que los objetivos deben ser precisados en colaboración con el paciente. La prescripción de medicamentos no siempre está indicada y su uso debe ser en general breve (de 5 a 7 días en una desintoxicación sin complicaciones) tanto ambulatoriamente como en hospitalización. La calidad de la relación médico-enfermo es esencial, así como establecer de entrada un apoyo psicoterapéutico o psicoterapias más estructuradas.

3) La calidad del seguimiento es el elemento esencial de la cura bio-psico-social.

Palabras clave: Alcoholismo, abstinencia, tratamiento, documento de consenso.

\section{Summary:}

Detoxification of the alcoholic makes sense only in a strategy that includes three stages:

1) Diagnosis: Although it is not easy in practice, it is essential to separate alcohol abuse from alcohol dependency particularly as, since 1994, the physical symptoms of abstinence are not necessary for the diagnosis.

2) Every confirmed case of alcoholic dependency justifies a complete and lengthy detoxification combined with a way of life project in which the objectives are established in collaboration with the patient. Prescribing medication is not always indicated and, in general, its use should be brief (from 5 to 7 days in detoxification without complications) for both outpatients and hospital patients. The quality of the doctor-patent relationship is essential as is the establishment of psychotherapeutic support or more structured psychotherapies from the very beginning.

3) The quality of the follow-up is the essential factor in the bio-psycho-social cure.

Key words: Alcoholism, withdrawal syndrome, treatment, consensus document.

opinión de expertos y de profesionales y en la realización de unas encuestas.

El primer paso sería elegir el tema tomando en consideración tres criterios fundamentales:

- La necesidad de responder a una preocupación de la Sanidad Pública, definida por su importancia en términos de frecuencia y/o de gravedad, el interés demostrado por sus profesionales, el impacto potencial sobre las prácticas.

- La disponibilidad de datos científicos de suficiente nivel publicados sobre el tema, para que el resultado pueda considerarse independiente de un único juicio subjetivo grupo coordinador de la conferencia

- La existencia de controversias susceptibles de ser clarificadas, e incluso zanjadas, gracias a las opiniones y a los datos aportados en un debate público. 
No hay duda de que estos tres requisitos se cumplen en el tema de la desintoxicación de los pacientes alcohólicos. El promotor, la Société FranÇaise d'Alcohologie, confió la responsabilidad de la conferencia a un comité organizador integrado por un grupo de personas especialmente interesadas en el tema y/o en la metodología. Este comité se encargó de definir las seis cuestiones prioritarias que debían responderse en relación con la desintoxicación de los alcohólicos. Asimismo, eligió un grupo coordinador que tuviera la misión de elaborar, al final de la conferencia, un texto consensual -las conclusiones y recomendaciones de dicha conferencia-, capaz de aportar una respuesta clara a cada una de las cuestiones planteadas. El grupo coordinador gozó de total libertad e independencia a la hora de redactar las mencionadas recomendaciones, y fue el único responsable directo del contenido y de la calidad de los textos elaborados.

Ese mismo grupo coordinador, constituido por personas especialmente sensibles al tema sin ser profesionales del mismo -lo que se consideró garantía de un cierto distanciamiento del problema-, contó con el siguiente asesoramiento:

- Un grupo bibliográfico cuya tarea era proporcionar un análisis objetivo de la literatura sobre el tema sin interpretar los resultados.

- Una serie de expertos encargados de reunir en un texto las informaciones (surgidas de su experiencia y de la literatura) que permitieran responder a una de las cuestiones planteadas.

- El público asistente a la conferencia, que podía intervenir al final de las sesiones, en el espacio dedicado a ruegos y preguntas.

Una difusión amplia de las recomendaciones surgidas en esta conferencia debería permitir una mejora en la calidad de los tratamientos ofrecidos a los alcohólicos, limitando su coste a lo estrictamente necesario, tanto en lo que se refiere a los medicamentos, sobre todo los sedantes, como a la hospitalización, gracias a una definición mucho más precisa de las indicaciones y de las modalidades del tratamiento de desintoxicación hospitalaria y ambulatoria.

A pesar de existir una disminución regular en el consumo medio de alcohol por año y por habitante desde 1970, Francia continúa siendo el tercer país europeo de mayor consumo, con la morbididad y la mortalidad que eso conlleva. El consumo abusivo de alcohol es la causa directa o indirecta de una de cada cinco consultas realizadas en medicina general (2), del 15 al $25 \%$ de las hospitalizaciones y de 30.000 a 35.000 fallecimientos al año, de los cuales en 1994 (3) 23.400 fueron debidos a casos de "alcoholismo crónico". Asimismo, es el origen de numerosos casos de violencia, suicidios, accidentes, etc...

Las modas en el consumo son muy variadas: abstinencia para una pequeña minoría, consumo habitual para la gran mayoría. Entre los consumidores habituales, lo más frecuente es utilizar el alcohol siguiendo unas pautas culturales y sociales que no revisten el menor peligro; sin embargo, una considerable minoría llega a crear un problema de salud pública. Entre ellos, dos millones y medio -llamados con frecuencia consumidores "de riesgo" o "abusivos"- pueden controlar aún su nivel de consumo, pero un millón y medio han perdido esa libertad: se trata de los alcohólicos. En la práctica, el límite entre estas dos últimas categorías no está siempre claro ni resulta fácil de determinar. En cualquier caso, ese modelo bidimensional de alcoholismo crónico, adoptado por la comunidad científica internacional (4), tiene el interés de introducir una indicación terapeútica; del diagnóstico de dependencia se deduce la necesidad de un proceso de desintoxicación terapeútico y de una abstinencia duradera, pues la cicatriz psicobiológica de la adicción ha sido objeto de pruebas experimentales. Si los consumidores abusivos no dependientes siguen un proceso de desintoxicación, su tratamiento no excluye el retorno eventual a un consumo moderado y controlado.

Siendo el consenso relativamente reciente en Francia, el alcoholismo ha pasado a ser considerado una enfermedad cuyo tratamiento exige la intervención médica y médico-social $(5,6)$. Su seguimiento es necesario, en primer lugar, por el sufrimiento individual, familiar y social que conlleva. Pero también resulta fundamental desde el punto de vista económico. Si analizamos los costes totales, los gastos ulteriores que pueden evitarse son muy superiores a los gastos derivados de una política de seguimiento (7).

Entre los numerosos especialistas que intervienen en dicho proceso, la ausencia o la falta de formación en el tema del alcoholismo, así como la no existencia de acuerdos, pueden generar -debido a la falta de diagnóstico o de indicaciones terapeúticas- graves complicaciones, fallecimientos, utilización abusiva de medicamentos prescritos más por la costumbre que por la existencia de datos científicamente establecidos. Un seguimiento terapeútico adaptado supone un diagnóstico precoz del alcoholismo, partiendo de unos datos clínicos, paraclínicos y, eventualmente, de la utilización de cuestionarios estandarizados.

El proceso de desintoxicación terapeútica debe integrarse dentro de una estrategia global de atención al alcohólico, ya que constituye una etapa ineludible y esencial. Asegura la prevención y el tratamiento de los síntomas de dependencia física y las complicaciones derivadas de la supresión repentina del alcohol. Asimismo, tiene como objetivo implicar al paciente en un proceso de atención continua, con la esperanza de obtener la prolongación de la abstinencia, a falta de una mejoría clínica a largo plazo. Momento de ruptura, favorece y refuerza la aceptación de la dependencia del alcohol, preludio de la reconquista de la autonomía 
y de la puesta en práctica de un nuevo proyecto existencial.

En Francia, es frecuente hospitalizar más o menos tiempo al paciente durante el proceso de desintoxicación(8). Debido a una serie de razones -más bien teóricas que económicas- estamos asistiendo al desarrollo reciente de alternativas ambulatorias. Éstas no se aplican ni en los casos de mayor gravedad -pueden aparecen peligrosas complicaciones del síndrome de abstinencia, crisis convulsivas, delirium tremens- ni en las patologías más complejas, con la presencia de comorbididades, nicotismo y toxicomanías ilegales en particular.

Actualmente, los métodos terapeúticos utilizados durante la desintoxicación son muy heterogéneos. Los tratamientos medicamentosos, no medicamentosos, las indicaciones de hospitalización, la duración óptima de los tratamientos adaptados a las diferentes formas clínicas de alcoholismo varían enormemente en función de los lugares, de las modas en uso, del tipo de formación de los equipos sanitarios (9).

Los estudios controlados se refieren fundamentalmente a las prescripciones medicamentosas (10-16), y dejan con frecuencia en la sombra, salvo algunas excepciones (17), tanto el contexto psicoterápico como el socioterápico, que la práctica ha confirmado hace mucho como indispensables para la ayuda del alcohólico.

En resumen, la disparidad de los métodos terapeúticos y de diagnóstico observados en la desintoxicación física del alcohólico exigen armonizar y racionalizar las estrategias. En la literatura internacional pueden hallarse síntesis y recomendaciones recientes $(13,14)$. Sin embargo, ninguna de ellas se ajusta a las prácticas francesas. Esta constatación ha justificado la celebración de una conferencia de consenso en París el 17 de marzo de 1999. Durante ésta, el grupo coordinador respondió a las seis cuestiones siguientes:

- ¿Cuáles son los enfoques conceptuales de la desintoxicación?

- ¿Cuáles son las indicaciones y las contraindicaciones de la desintoxicación?

- ¿Cuáles son las modalidades de desintoxicación?

- ¿Qué modalidades prácticas proponer cuando el alcoholismo está asociado a una o varias sustancias psicoactivas?

- ¿Qué modalidades prácticas proponer en caso de asociación a otras patologías?

- ¿Qué tratamiento proponer ante un accidente en la desintoxicación?

\section{CUESTION 1 \\ ¿CUÁLES SON LOS ENFOQUES CONCEPTUALES DE LA DESINTOXICACIÓN?}

\section{La noción de desintoxicación y su relación con el alcoholismo:}

- Desintoxicación: abandono del consumo de alcohol por parte del alcohólico (accidental, por iniciativa propia o inscrita en una perspectiva terapeútica).

- Síndrome de abstinencia: manifestaciones sintomáticas que hacen su aparición de forma inmediata o diferida durante los diez días que siguen a dicha interrupción del consumo. Estas manifestaciones reflejan estados de insuficiencia psíquica, comportamental y física.

- Alcoholismo: las clasificaciones internacionales más recientes han dejado de considerar los síntomas físicos que se producen tras la interrupción del consumo como indispensables en el diagnóstico de alcoholismo que tiene, asimismo, otras manifestaciones:

Psíquicas: deseo compulsivo de beber alcohol; el individuo es incapaz de controlar su consumo.

\section{Comportamentales:}

- El afán de consumir alcohol va imponiéndose sobre la mayoría de las actividades del individuo.

- Fenómeno de prevención: el individuo consume alcohol, a menudo desde por la mañana, con la intención de prevenir o de suprimir los síntomas producidos por el no-consumo.

- Fijación progresiva de las modalidades de consumo de alcohol, dictada por la necesidad de mantener una alcoholemia suficiente.

- Aumento de la tolerancia, que conduce al individuo a consumir cada vez mayores cantidades de alcohol.

Desintoxicación y alcoholismo: los individuos adictos al alcohol deben contar con ayuda terapeútica para lograr desintoxicarse.

\section{Descripción del síndrome de abstinencia}

El síndrome de abstinencia se asocia en mayor o menor medida con distintos tipos de manifestaciones.

En la mayoría de los casos se trata de desarreglos:

- subjetivos: ansiedad, agitación, irritabilidad, insomnio, pesadillas

- neurovegetativos: sudores, taquicardia, hipertensión arterial, temblores

- digestivos: anorexia, nauseas, vómitos

El cuadro puede complicarse durante las siguientes horas con la aparición de:

- signos de confusión

- alucinaciones 
- convulsiones

- hipertermia

El síndrome desaparece, de forma espontánea o con la ayuda de un tratamiento, en un plazo de dos a cinco días.

La aparición más tardía o la persistencia de estos desarreglos una vez transcurrido dicho período deben hacer pensar en otras adicciones asociadas, especialmente a las benzodiacepinas (BZD).

\section{Objetivo de una desintoxicación terapeútica}

Su finalidad es entrar en un proceso de abstención completo y duradero. Debe ponerse en práctica siempre que haya adicción al alcohol, con independencia de que existan o no manifestaciones físicas.

Una vez que el individuo crea adicción a una sustancia, son numerosos los expertos que consideran muy difícil, por no decir imposible, el retorno a un consumo moderado.

Para conseguir una desintoxicación de buena calidad deben reunirse una serie de circunstancias:

- La existencia de unas condiciones óptimas de comodidad y de seguridad que permitan prevenir o tratar el síndrome de abstinencia.

- Asegurar un seguimiento terapeútico prolongado en una institución o ambulatorio.

- Ayudar al paciente a convertir la desintoxicación en una experiencia positiva a largo plazo, tanto en el plano personal, como en el familiar y profesional.

\section{Criterios de evaluación del síndrome de abstinen- cia y del alcoholismo}

Los signos clínicos continúan siendo el criterio esencial de evaluación.

Las escalas propuestas para evaluar el síndrome de abstinencia y el alcoholismo son muy numerosas. Aunque no podemos subestimar el interés epidemiológico, pedagógico e investigador de las mismas, muy pocas resultan válidas en nuestro país.

\section{Recomendaciones}

Todo síndrome de adicción al alcohol justifica el abandono total y prolongado de su consumo. Éste debe integrarse en un proyecto cuyos objetivos queden acordados con el propio paciente.

Resulta primordial que cualquier propuesta de desintoxicación alcohólica tenga en cuenta al individuo que padece el problema, quien debe ser consultado e informado. Su aprobación es necesaria para poner en marcha el proyecto terapeútico.

\section{CUESTIÓN 2 \\ ¿CUÁLES SON LAS INDICACIONES Y CONTRAIN- DICACIONES DE LA DESINTOXICACIÓN?}

Se recomienda una desintoxicación terapeútica a todas aquellas personas con problemas de alcoholismo.

\section{Indicaciones}

- Abandono programado del consumo de alcohol

La desintoxicación es una etapa necesaria, entre el antes y el después, en todo proyecto terapeútico. Debe contar con el apoyo de un equipo pluridisciplinario.

¿Quién debe colaborar?

- la familia

- los amigos

- los distintos especialistas médico-sociales

- el propio enfermo

La elección de iniciar la desintoxicación está en función del deseo del alcohólico, de su necesidad de cambio y de su sufrimiento físico y psicológico.

¿Cuál sera su proyecto de vida?

La petición de ayuda para desintoxicarse supone un mínimo de elaboración.

Todo proyecto terapeútico debe tener en cuenta la motivación, la implicación y las capacidades del paciente.

¿En qué momento?

El momento de empezar a vivir sin alcohol debe ser decidido conjuntamente por el paciente y por el médico cuando tanto el contexto como la madurez del proyecto personal del paciente garanticen su éxito.

- Abandono del alcohol no programado

Ello supone la interrupción obligada del consumo:

- Puede ayudar al alcohólico a darse cuenta de que necesita seguir un tratamiento.

- En medios carcelarios, la interrupción brusca y repentina del consumo aumenta el riesgo de accidentes entre los alcohólicos, a menudo adictos a varias sustancias.

\section{Recomendaciones}

El grupo coordinador recomienda:

- Una evaluación de los resultados y del interés del tratamiento.

- La formación de un equipo de asistencia siguiendo la escala DETA y la creación de espacios de asistencia para los alcohólicos en medios carcelarios.

- La investigación sistemática de una posible adicción al alcohol desde el ingreso en prisión. 
El síndrome de abstinencia en los servicios de urgencia

Es necesario detectar sistemáticamente el síndrome de abstinencia en todas las hospitalizaciones de urgencia.

\section{Recomendaciones}

Establecer un primer contacto haciendo referencia a la salud y el alcohol antes de que el paciente abandone el centro.

- Desintoxicaciones múltiples

A veces el paciente alcohólico vuelve a tener problemas de adicción después de haber seguido un tratamiento de desintoxicación. Es aconsejable no responder sistemáticamente a todas sus demandas y trabajar con él para hacer madurar su motivación.

Estas desintoxicaciones múltiples deben inscribirse en un proceso de seguimiento a largo plazo.

- Las especificidades del abandono del alcohol

Las mujeres alcohólicas presentan a menudo un consumo asociado a los psicótropos. En caso de comorbididad depresiva, se recomienda la puesta en marcha de un tratamiento especializado desde el inicio de la desintoxicación.

\section{Mujeres embarazadas.}

El riesgo de malformaciones en el recién nacido justifica la indicación imperativa de una desintoxicación terapeútica.

Niños y adolescentes:

No están especificamente codificadas las formas de desintoxicación terapeútica. La investigación de trastornos psicopatológicos y de poliadicciones debe ser sistemática.

\section{Recomendaciones}

He aquí las propuestas del grupo coordinador:

- Investigación de prácticas adaptadas a la desintoxicación terapeútica de los adolescentes.

- Puesta en marcha de un seguimiento terapeútico asociado a una idea socioeducativa.

\section{Individuos de más de 65 años:}

Se recomienda efectuar un seguimiento terapeútico específico dentro de grupos de edades homogéneas.

\section{Individuos en situación de precariedad:}

Existe un fuerte consenso a la hora de considerar que el aislamiento y la falta de inserción social requieren una hospitalización.

Los enfermos que padecen graves patologías como cáncer, hepatitis C, SIDA, etc... necesitan interrumpir el consumo de alcohol siguiendo un tratamiento terapeútico específico.

\section{Contraindicaciones}

No existen contraindicaciones absolutas.

A pesar de ello, no se recomienda la desintoxicación inmediata en los siguientes casos:

- no demanda por parte del alcohólico.

- ausencia de un proyecto terapeútico y social.

- situaciones de crisis (afectiva, social, profesional)

Ello no impide el tratamiento y el seguimiento del paciente con la perspectiva de una desintoxicación posterior.

\section{CUESTION 3 ¿CUÁLES SON LAS FORMAS DE DESINTOXICA- CIÓN?}

Para abandonar el consumo de alcohol es necesario seguir un tratamiento médico que cuente con distintos apoyos previamente concertados. Para asegurar la calidad y la seguridad del proceso de desintoxicación es fundamental la formación de todos aquellos que intervienen en el proceso.

Un alcohólico puede desintoxicarse en un ambulatorio o en una estructura de hospitalización ("desintoxicación institucional").

- Ventajas de la desintoxicación ambulatoria: no interrupción de la actividad profesional; mantenimiento de las relaciones familiares y sociales; mayor aceptación, ya que no obliga a asumir socialmente el estatus de enfermo; compatibilidad con un síndrome de abstinencia moderado; participación del paciente y de su entorno.

- Ventajas de la desintoxicación institucional: vigilancia continua (personas aisladas); enfermo alejado de su entorno (alcohol, situaciones de riesgo o conflictivas); garantía de la realidad del abandono del alcohol y de la observancia de las prescripciones; indicación formal en caso de patologías complejas o de síndrome de abstinencia grave.

\section{Lugares de tratamiento}

- La desintoxicación ambulatoria presenta contraindicaciones (10 al 30\% de los enfermos): alcohológicas -fuerte adicción física; antecedentes de delirium tremens o de crisis convulsiva generalizada-; somáticas; psiquiátricas; socioambientales.

- Cuando la desintoxicación es institucional, los estudios no demuestran que sea beneficioso continuar el ingreso una vez transcurridos diez días, excepto en caso de existir complicaciones somáticas o psiquiátricas.

- Un tratamiento preventivo a base de BZD reduce la frecuencia de los accidentes del 10\% a menos del 
$2 \%$. El interés de un tratamiento medicamentoso de desintoxicación en los enfermos que no presentan signos físicos de dependencia no ha sido demostrado. El pronto inicio del tratamiento es un elemento esencial de la eficacia preventiva. En ambulatorio, sin la posibilidad de una evaluación a priori del riesgo y sin existir un seguimiento cercano, la prevención medicamentosa ha de ser sistemática. En institución, pueden adoptarse dos posturas diferentes: prescripción sistemática o adaptación en función del cuadro clínico inicial y de su evolución.

\section{Recomendaciones}

La desintoxicación terapeútica precisa un seguimiento médico y pluridisciplinario de acuerdo con la red terapeútica.

El grupo coordinador recomienda plantearse en primer lugar una desintoxicación ambulatoria, respetando en todo momento las contraindicaciones.

La prevención medicamentosa de los accidentes debe ser sistemática cuando el tratamiento de desintoxicación es ambulatorio.

Las indicaciones y los resultados de las hospitalizaciones prolongadas deben ser evaluadas por estudios complementarios.

\section{El tratamiento psicosocial durante la desintoxica- ción tiene tres funciones esenciales:}

La información, el apoyo y el consuelo, dentro de un entorno tranquilo, abierto y confiado, juegan un importante papel en la prevención y en el control de los síntomas y accidentes en la desintoxicación. Ello permite disminuir las dosis de la medicación. Las técnicas de relajación y de readaptación corporal merecen ser valoradas.

El establecimiento de una relación terapeútica que debe continuar más allá de la desintoxicación tiende a aumentar la autoestima y a restaurar el narcisismo. Dependiendo de los pacientes, la forma de terapia puede ser individual, de grupo, familiar o de pareja, comportamental-cognitiva. En un estudio realizado al azar, la intervención de movimientos de ayuda mutua durante la desintoxicación mejoró significativamente el pronóstico a largo plazo.

El apoyo social completa el tratamiento médico y el acercamiento psicológico en una perspectiva de readaptación, fundamentalmente en las poblaciones en situación de precariedad.

\section{Tratamientos medicamentosos}

- Los psicótropos

Las benzodiacepinas (BZD) son en nuestros días el tratamiento medicamentoso más utilizado para el sín- drome de abstinencia del alcohólico. Reducen la incidencia y la gravedad de dicho síndrome, las crisis epilépticas y de delirium tremens (DT). Las BZD de vida media larga como el diazepam previenen mejor las crisis epilépticas, pero aumentan el riesgo de acumulación en caso de insuficiencia hepatocelular. El oxazepam tiene, así, un interés especial por no modificar el metabolismo. Las BZD de vida media corta presentan un potencial de abuso más alto.

- Guía práctica de la desintoxicación a base de BZD: Debe utilizarse preferentemente la vía oral.

Tres esquemas de prescripción son posibles:

Prescripción de dosis repartidas en 24 horas:

- Un comprimido de 10 mg de diazepam cada seis horas durante los cuatro primeros días; ir reduciendo la dosis del cuarto al séptimo día hasta prescindir por completo de esta medicación.

O bien:

- Seis comprimidos de 10 mg de diazepam el primer día; ir reduciendo un comprimido al día hasta interrumpir su toma.

Se recomienda esta estrategia en ambulatorio.

Prescripción personalizada: eventualmente guiada por una escala que evalúe la gravedad de los síntomas.

Utilización de una dosis de carga oral de una BZD de vida media larga.

Otras BZD diferentes al diazepam pueden ser utilizadas con análoga eficacia. Se calcula que $10 \mathrm{mg}$ de diazepam equivalen a $30 \mathrm{mg}$ de oxazepam, $2 \mathrm{mg}$ de lorazepam, $1 \mathrm{mg}$ de alprazolam y 15 mg de cloracepate.

\section{- Otros psicótropos}

El meprobanate, el tetrabamate, los barbitúricos y los neurolépticos tienen una relación beneficio-riesgo inferior a los de las BZD.

\section{- Los tratamientos asociados}

La hidratación: las aportaciones hídricas deben ser suficientes pero sin hiperhidratación. Han de evitarse las perfusiones en el enfermo consciente.

La prescripción de magnesio sólo debe contemplarse en casos de hipocalemia.

La tiamina: el abandono del consumo puede favorecer la aparición de graves problemas neurológicos o cardíacos debido a la carencia de tiamina, especialmente en caso de aporte asociado de glucosa.

Es necesario administrar tiamina (500 gr diarios) a todo aquel paciente que inicie una desintoxicación. La administración parenteral, preferiblemente por perfusión intravenosa, debe ser preconizada en caso de existir signos clínicos de carencia durante la primera semana. Ninguno de los métodos terapeúticos no 
convencionales, también llamados alternativos, ha demostrado ser de interés.

\section{Recomendaciones}

La prescripción medicamentosa no debe hacer olvidar la importancia fundamental del tratamiento psicológico y social.

Los movimientos de ayuda mutua han de producirse durante el proceso de desintoxicación.

Las BZD son los medicamentos más utilizados en el tratamiento preventivo del síndrome de abstinencia.

La duración del tratamiento con BZD, prescritas en dosis decrecientes, no debe exceder los siete días, excepto en caso de existir complicaciones.

Es necesario desarrollar la prescripción personalizada. La utilización de escalas evaluadoras de la gravedad de los síntomas, que deben validarse en francés, puede resultar de gran ayuda.

La hidratación debe ser oral en el enfermo consciente.

La tiamina debe prescribirse sistemáticamente de forma preventiva (por vía parenteral en caso de signos clínicos de carencia).

\section{CUESTION 4}

\section{¿QUÉ MODALIDADES PRÁCTICAS PROPONER CUANDO EL ALCOHOLISMO ESTÁ ASOCIADO A LA ADICCIÓN A UNA O VARIAS SUSTANCIAS PSI- COACTIVAS?}

Comprometerse a un proceso de desintoxicación en caso de adicción a varias sustancias psicoactivas es un trabajo largo e intensivo. La gravedad de los síntomas, la complejidad de las situaciones, la frecuencia de los problemas psiquiátricos subyacentes obligan a menudo a la hospitalización en un centro especializado.

Las estrategias terapeúticas son más difíciles de elaborar. Generalmente es inevitable dar prioridad al tratamiento de una adicción sobre las demás.

No existen datos sobre la asociación alcohol-cannabis.

\section{Alcohol y tabaco}

Entre el 80 y el $95 \%$ de los individuos alcohólicos son también fumadores. El incremento del índice de mortalidad que genera esta doble dependencia es superior a la que se atribuye por separado al tabaco o al alcohol.

Es muy poco frecuente que los especialistas ofrezcan ayuda para dejar el tabaco. Las razones aducidas son diversas: no comprometer el proceso de desinto- xicación alcohólica, la dependencia a la nicotina está socialmente bien aceptada y, a pesar de ser un error, no se considera peligrosa... Esta actitud se observa sobre todo entre los especialistas fumadores.

Actualmente, tanto especialistas como enfermos conceden cada vez mayor importancia a la necesidad de efectuar las desintoxicaciones del alcohol y del tabaco al mismo tiempo. Dos modalidades prácticas son posibles:

- Una desintoxicación alcohólica asociada a una sustitución de nicotina que permita diferir la desintoxicación de tabaco.

- Una terapeútica común a ambas con clonidina. Sus efectos secundarios justifican que el tratamiento se efectúe en un medio hospitalario.

Ningún estudio está a favor de una evolución desfavorable del alcoholismo tras el abandono del tabaco.

\section{Recomendaciones}

El grupo coordinador subraya la necesidad de:

- Tomar en consideración el tabaquismo precoz y reforzar las acciones de información sobre el peligro que conlleva consumir sustancias psicoactivas, especialmente entre los adolescentes.

- Proponer una desintoxicación simultánea en cuanto el estado de motivación de los pacientes lo permita, lo que supone abordar sin demora la información y las formas de desintoxicación de tabaco.

El grupo coordinador subraya la importancia de la motivación y recomienda reforzar en el paciente la confianza en sí mismo, así como su capacidad para conseguir una doble desintoxicación.

\section{Alcohol y benzodiacepinas}

El consumo concomitante de alcohol y de BZD es susceptible de generar graves problemas de comportamiento. El alcohólico debe considerarse un individuo de riesgo en relación a la dependencia de las BZD.

La utilización de BZD para tratar el síndrome de abstinencia en aquellos pacientes ya dependientes plantea diversas cuestiones: elección de la posología, mantenimiento de la molécula consumida o prescripción de otra BZD.

\section{Recomendaciones}

Es necesario excluir toda desintoxicación brutal y saber admitir un proceso más largo y complejo a dos tiempos: primero alcohol, después BZD.

El grupo coordinador recomienda no prescribir BZD a un alcohólico que continúe bebiendo.

El grupo coordinador propone en primer lugar: mantenimiento de idéntica molécula y posología durante la fase inicial de desintoxicación alcohólica (siete días). Una vez finalizada esta etapa, la posología 
de BZD debe reducirse un $25 \%$ gradualmente cada tres días.

Otras estrategias pueden hacerse necesarias en función de la situación clínica: aumento de la posología, cambio de molécula a favor de una BZD de vida media larga.

La utilización de medicamentos neurolépticos sedativos está desaconsejada a priori debido a la existencia de un riesgo mayor de convulsiones.

\section{Alcohol y opiáceos}

En el individuo sometido a un tratamiento de sustitución, la desintoxicación alcohólica es posible; sin embargo, el riesgo de que se produzca una depresión respiratoria por la asociación de la metadona o bupremorfina con las BZD hace necesario extremar la vigilancia.

\section{Recomendaciones}

La desintoxicación simultánea del alcohol y de la heroína exige una hospitalización.

Al advertir el incremento de los consumidores adictos a varias sustancias: alcohol, opiáceos (heroína o productos de sustitución) y BZD, el grupo coordinador resalta la necesidad de realizar estudios sobre el tema (¿Incidencia del alcoholismo? ¿Interacciones farmacodinámicas y farmacocinéticas? ¿Pronósticos psicopatológicos?)

\section{Alcohol y cocaína}

Esta adicción simultánea es frecuente. No existe ningún tratamiento codificado para su desintoxicación. La utilización de BZD no ha demostrado ser positiva.

\section{Recomendación}

Necesidad de efectuar estudios sobre las complicaciones psicopatológicas de esta asociación.

Es indispensable que el tratamiento de los pacientes polidependientes vaya acompañado de una psicoterapia y de un apoyo socioeducativo.

\section{CUESTIÓN 5 \\ ¿QUÉ MODALIDADES PRÁCTICAS PROPONER EN CASO DE ASOCIACIÓN CON OTRAS PATOLOGÍAS?}

\section{Patologías psiquiátricas}

- Ansiedad

Hay tres causas posibles:

- Ligada a la desintoxicación.

- Inducida por la adicción.

- Patologías psiquiátricas (fobias, cuadros de pánico, ansiedad generalizada), con menor frecuencia,

\section{Recomendación}

El tratamiento de la ansiedad durante la desintoxicación exige una vigilancia regular del paciente y la prescripción eventual de un ansiolítico adaptado a la severidad del cuadro. Es mayor el nivel de eficacia de las BZD que de los carbamatos. En ambulatorio, las prescripciones no excederán una semana.

El tratamiento de un cuadro de ansiedad primario puede hacerse coincidir con la desintoxicación o efectuarse inmediatamente después, dependiendo de las modalidades admitidas por cada una de sus formas clínicas.

\section{- Depresión}

Es frecuente la aparición de síntomas depresivos durante la desintoxicación; la mayor parte de ellos remiten entre las dos y las cuatro semanas. No obstante, entre el 10 y el $15 \%$ de los casos el cuadro es más severo y puede llegar a incluir características melancólicas.

El riesgo de suicidio está siempre presente. El grupo coordinador subraya que los determinantes del riesgo de suicidio del alcohólico no van ligados únicamente a la depresión.

\section{Recomendaciones}

Se aconseja esperar un mínimo de dos a cuatro semanas de desintoxicación antes de introducir un tratamiento antidepresivo.

Debe replantearse esta espera si existe un recrudecimiento del síndrome depresivo que aumente el riesgo de suicidio.

Los antidepresivos serán prescritos, cuando estén indicados, siguiendo las mismas posologías y la misma duración que en las demás formas de depresión. Las psicoterapias se adaptarán a la naturaleza del cuadro que origina la depresión.

\section{- Alcoholismo y problemas psicóticos}

Todas las psicosis pueden ir asociadas al alcoholismo o a una conducta de abuso del alcohol.

\section{Recomendaciones}

La asociación de una psicosis y de una adicción al alcohol exige el tratamiento integrado de los dos problemas.

- Trastornos de personalidad

Algunos trastornos previos de la personalidad pueden complicar el tratamiento, especialmente a nivel social.

\section{Recomendaciones}

Es necesario realizar estudios que determinen con mayor exactitud su naturaleza y ayuden a mejorar su tratamiento. 


\section{Patologías somáticas}

- Patologías hepáticas de origen alcohólico o viral.

La desintoxicación tiene un efecto favorable sobre estas patologías, tanto si son de origen alcohólico como viral B o C.

- Pancreatitis crónica alcohólica

Justifica una desintoxicación.

- Desórdenes nutricionales y neuropatías

Malnutrición, encelopatías carenciales

La encefalopatía de Gayet-Wernicke debe prevenirse prescribiendo sistemáticamente vitamina B1 (cf. Q3).

Se trata de una urgencia metabólica cuyo tratamiento es $1 \mathrm{~g}$ diario de tiamina por vía intravenosa.

Hiponatremia grave

La hiponatremia profunda o acompañada de signos neurológicos hace necesaria la hospitalización.

Su tratamiento debe ser progresivo, basado en la restricción hídrica y en un aporte isotónico.

Polineuritis y miopatía alcohólica

Pueden mejorar con la abstinencia y una nutrición adecuada.

- Complicaciones cardiovasculares

El abandono del consumo de alcohol puede mejorar la cardiomiopatía alcohólica y disminuir el riesgo de los problemas de ritmo.

La hipertensión arterial, frecuente entre los alcohólicos, deber ser vigilada.

- Interacciones de medicamentos

La desintoxicación favorece la acumulación de medicamentos e invita a ajustar las dosis.

- Patología quirúrgica intercurrente y desintoxicación accidental

El no reconocimiento de la adicción al alcohol puede originar un síndrome de abstinencia más agudo y posibles infecciones post-operatorias.

\section{Recomendaciones}

- La desintoxicación forma parte integrante del tratamiento de las complicaciones somáticas del alcohol.

- En caso de insuficiencia hepatocelular o de cirrosis, la eventual prescripción de BZD deber ser muy prudente y realizarse en un centro especializado.

- Mientras se realiza la desintoxicación, han de tenerse presentes las adaptaciones posológicas de los medicamentos.

- En medios quirúrgicos, el grupo coordinador recomienda la investigación pre-operatoria de una adicción al alcohol, así como los signos precursores de los accidentes neurológicos de la desintoxicación en el post-operatorio (cf Q6).

\section{CUESTIÓN 6 \\ ¿QUÉ TRATAMIENTO PROPONER ANTE UN ACCI- DENTE EN LA DESINTOXICACIÓN?}

\section{Introducción}

Las crisis epilépticas y el delirium tremens (DT) son sus manifestaciones. Debe abandonarse el término pre-DT, fuente de confusión, en beneficio de una clara delimitación de los distintos niveles de gravedad del síndrome de abstinencia.

Estos accidentes sobrevienen cuando el abandono del consumo de alcohol es impuesto o se produce algún error en el transcurso de una desintoxicación programada.

Ellos justifican en todos los casos, dependiendo de su gravedad, la hospitalización en una unidad de cuidados intensivos o en una unidad médica convencional.

Un proyecto de seguimiento médico de la desintoxicación se ofrece al finalizar dicha hospitalización.

\section{Delirium tremens}

- El tratamiento psicótropo se basa en la utilización de BZD por vía intravenosa. El diazepam y el flunitrazepam han probado su eficacia. Se emplea preferentemente el diazepam, administrando una dósis de $10 \mathrm{mg} /$ hora. El midazolam intravenoso también se utiliza con frecuencia en la práctica, si bien no ha sido validado.

- El riesgo de depresión respiratoria exige la hospitalización en una estructura que disponga tanto de medios de reanimación como el antídoto necesario (flumazenil). El resto de los psicótropos -tiapride, fenotiacina, meprobamate, clometiazole, barbitúricos- no pueden recomentarse en estos casos. El haloperidol se utiliza asociado a las BZD en caso de síndrome alucinatorio persistente.

- El reequilibrio hidroelectrolítico es indispensable, con la seguridad de un chequeo paraclínico inicial y repetido. Las aportaciones hídricas (por vía parenteral) buscan corregir la deshidratación sin excesos y reestablecer la diuresis. La administración añadida de $500 \mathrm{mg} / \mathrm{j}$ de tiamina está indicada en caso de aporte de glucosa.

- La hipertermia debe ser corregida.

- El tratamiento de hipocalemia necesita la aportación conjunta de potasio y de magnesio.

- La clonidina puede tener interés en el tratamiento de un acceso de hipertensión grave.

- El tratamiento etiológico debe ser aplicado conjuntamente en todos los casos.

\section{Crisi(s) convulsiva(s)}

Deben estudiarse conjuntamente las restantes etiologías frecuentes en el alcoholismo. 
- Hipoglucemia, hiponatremia

- Traumatismo craneal

- Infección cerebral-meníngea

- Accidente vascular-cerebral

- Intoxicación medicamentosa

- Epilepsia alcohólica

- Las convulsiones en la desintoxicación

- Afectan a los individuos de riesgo (consumo de psicótropos, antecedentes convulsivos, antecedentes de traumatismo craneano)

- El 90\% aparecen en las 48 primeras horas

- Aparecen crisis de Gran Mal en el 95\% de los casos

- Se repiten a menudo con pareja intensidad durante 12 horas

- No conllevan anomalías electroencefalográficas

\section{- El tratamiento}

Las convulsiones independientes de la desintoxicación son tratadas específicamente.

Una crisis convulsiva a causa de la desintoxicación no justifica un tratamiento antiepiléptico. Cuando las crisis se reproducen, la utilización de diazepam o clonazepam por vía intravenosa queda justificada siempre que se disponga de medios de reanimación respiratoria. En caso contrario, debe vigilarse estrechamente al paciente hasta la llegada de medios de transporte medicalizados.

La recuperación de la conciencia autoriza la administración de una BZD per os, desaconsejándose la vía intramuscular.

La calidad metodológica insuficiente de la mayoría de los estudios realizados no permite formular recomendaciones de grado $A$.

\section{BIBLIOGRAFÍA}

1. Les conférences de consensus. Base méthodologique pour leur réalisation en France. ANDEM, 1997, 48 p.

2. HUAS D, DARNE B, RUEFF B, LOMBRAIL P, LEBLANC B. "Malades alcooliques " et consultations en Médecine Générale : prévalence et détection. Rev Prat (Med Gen), 1990, № 81, 45-49.
3. La Santé en France 96. Haut Comité de la Santé Publique, La Documentation Française, 1996, 24-27.

4 Substance-related disorders. Diagnostic and statistical manual of mental disorders. Fourth edition. American Psychiatric Association, 1994, 175-272.

5. SOURNIA JC. L'histoire de l'alcoolisme. Flammarion, Paris, 1986, $322 \mathrm{p}$.

6. TAKALA JP, LETHO J. Finland: the non medical model reconsidered. En : Cure, care or control-Alcoholism treatment in sixteen countries. KLINGEMANN $\mathrm{H}$, TAKALA JP, HUNT G eds, New York : State University of Nzw York Press, 1992, 87-110.

7. MOSSE P. L'économie du sevrage de l'alcoolique : une méthode comptable, des enjeux institutionnels. Alcoologie, 1999, suppl. 2 (en prensa).

8. LE BARS Y, ORCEL S, BALMES JL. Prise en charge des syndromes de sevrage alcoolique en milieu hospitalier. Enquête Nationale. Alcoologie 1999 (en prensa).

9. SAITZ R, MAYO-SMITH MF, FRIEDMAN LS, CALKINS DR. Nationwide practice patterns in the treatment of alcohol withdrawal. Clin Res, 1993, 41, 549A.

10. GUTHRIE SK. The treatment of alcohol withdrawal. Pharmacotherapy, 1989, 9, 131-143.

11. LISKOW BI, GOODWIN DW. Pharmacological treatment of alcohol intoxication, withdrawa] and dependence : a critical review. J Stud Alcohol, 1987, 48, 356-370.

12. RO54ACH MK, SELLERS EM. Management of the alcohol withdrawal syndrome. Annu Rev Med, 1991, 42, 323-340.

13. MAYO-SMITH MF. Pharmacological management of alcohol withdrawal a meta-analysis and evidence-based practice guideline. JAMA, 1997, 278, 144-151.

14. WILLIAMS D, Mc BRIDE AJ. The drug treatment of alcohol withdrawal symptoms : a systematic review. Alcohol, 1988, 33, 103-115.

15. SULLIVAN JT, SWIFT RM, LEWIS DC. Benzodiazepine requirements during alcohol withdrawal syndrome : clinical implications of using a standardized withdrawal scale. J Clin Psychopharmacol, 1991, 11, 291-295.

16. SAITZ R, MAYO-SMITH MF, ROBERTS MS, REDMOND HA, BERNARD DR, CALKINS DR. Individualized treatment for alcohol withdrawal. A randomized doubleblind controlled-trial. JAMA, 1994, 272, 519-523.

17. NARANJO CA, SELLERS EM, CHATER K, IVERSEN P, ROACH C, SYKORA K. Non pharmacologic intervention in acute alcohol withdrawal. Clin Pharmacol Ther, 1983, 34, 214-219. 